\section{Cureus}

Received 01/13/2016

Review began 01/22/2016

Review ended 02/01/2016

Published 02/12/2016

\section{C) Copyright 2016}

Graff et al. This is an open access article distributed under the terms of the Creative Commons Attribution License CC-BY 3.0., which permits unrestricted use, distribution, and reproduction in any medium, provided the original author and source are credited.

\title{
Gallbladder Carcinoma, the Difficulty of Early Detection: A Case Report
}

Andrew E. Graff ${ }^{1}$, Stephen L. Lewis ${ }^{1}$, Jonathan R. Bear ${ }^{1}$, David C. Van Echo ${ }^{2}$, Hugh M. Dainer $^{3}$

1. Radiation Oncology, Walter Reed National Military Medical Center 2. Hematology/Oncology, Walter Reed National Military Medical Center 3. Diagnostic Radiology, Walter Reed National Military Medical Center/Uniformed Services University of Health Sciences

$\square$ Corresponding author: Andrew E. Graff, zxga@yahoo.com

Disclosures can be found in Additional Information at the end of the article

\section{Abstract}

Gallbladder carcinoma (GBC) is an uncommon malignancy with a high mortality rate. Detecting gallbladder carcinoma in its early stages can be difficult, despite improvements in ultrasound and computed tomography (CT) imaging. Most diagnoses of GBC are made at advanced stages, with the majority being found incidentally during surgery for cholelithiasis. The presented case demonstrates the difficulty of diagnosing GBC preoperatively in its early stages.

Categories: Medical Education, Radiology, Oncology

Keywords: gallbladder, gallbladder cancer, carcinoma, cancer, cholecystectomy, gallbladder carcinoma

\section{Introduction}

Gallbladder carcinoma (GBC) is a rare malignancy, but the most common malignancy of the biliary tract. The incidence of GBC in the US is 1.2 per 100,000 . The majority of gallbladder carcinomas are diagnosed incidentally, usually when exploring for cholelithiasis [1]. Most diagnoses of GBC are made at advanced stages and have been associated with poor outcomes [2]. Several risk factors have been identified for GBC. The leading risk factors include female gender, cholelithiasis, and advancing age. Gallbladder polyps, porcelain gallbladder, congenital biliary cysts, abnormal pancreaticobiliary duct junction, and carcinogen exposure have all been associated with GBC. Additional reported risk factors include smoking, obesity, diabetes, chronic infections (Salmonella, Helicobacter), and medications (such as methyldopa, OCPs, isoniazid, and estrogen) [3-5]. Herein, we present a case of locally advanced GBC with nodepositive disease found during laparoscopy for acute cholecystitis.

\section{Case Presentation}

A 65-year-old obese, African-American female with a history of hyperlipidemia presented to her primary care physician (PCP) in May 2013 for right neck pain radiating to her right shoulder for several weeks. The patient's initial blood work revealed an elevation in her liver enzymes; otherwise, all other lab results were normal. Additionally, a right upper quadrant (RUQ) ultrasound was performed which demonstrated probable gallbladder sludge and stones, with a low likelihood of being polyps or gallbladder neoplasm given the absence of discrete vascularity (Figures 1,2). 


\section{Cureus}

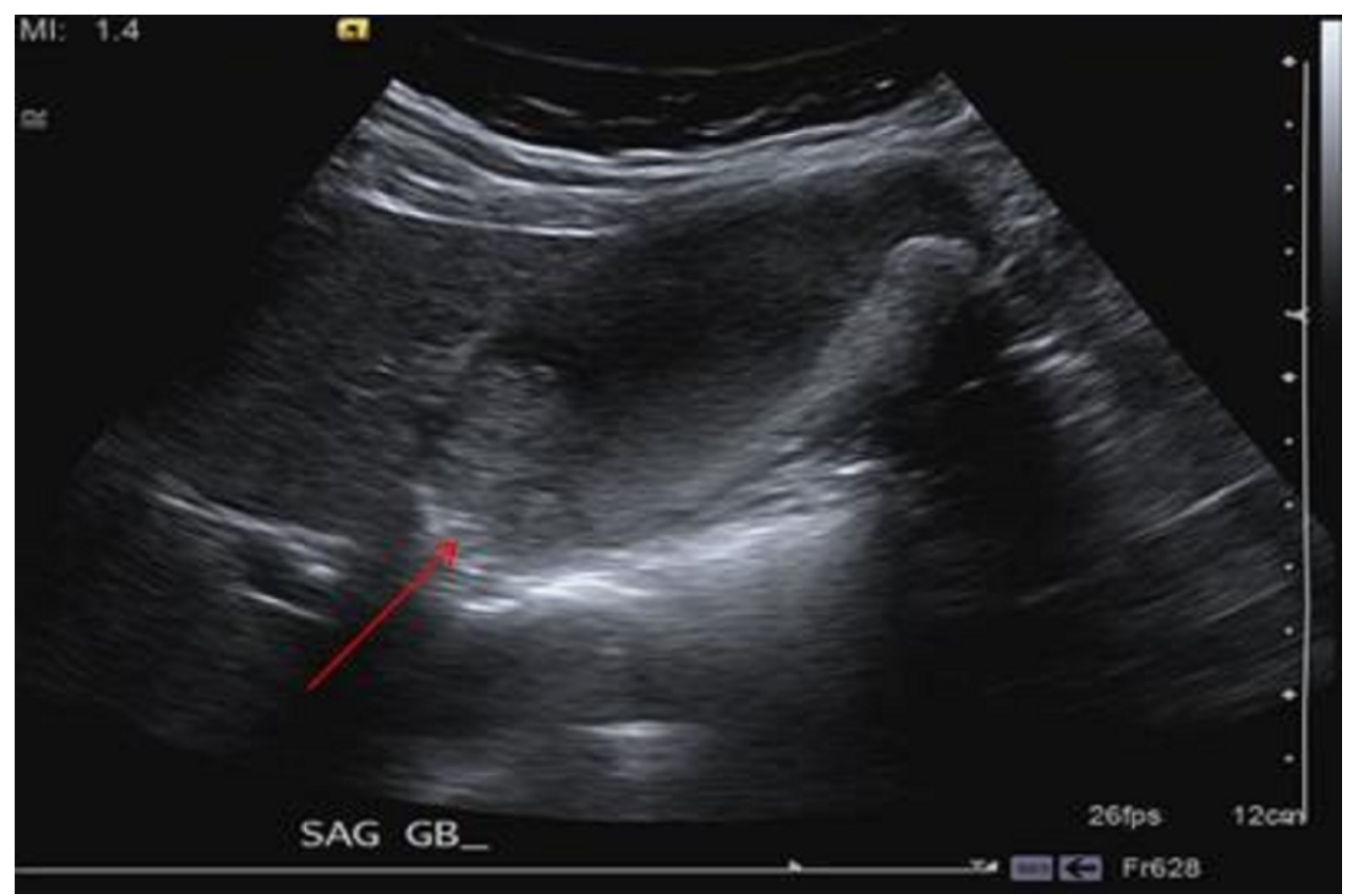

\section{FIGURE 1: Initial right upper quadrant ultrasound.}

The image demonstrates intraluminal hypoechoic (red arrow) material. Given the appearance and lack of discrete internal flow, it appears to be most likely due to gallstones or tumefactive sludge, which is a benign mimic of a neoplasm.

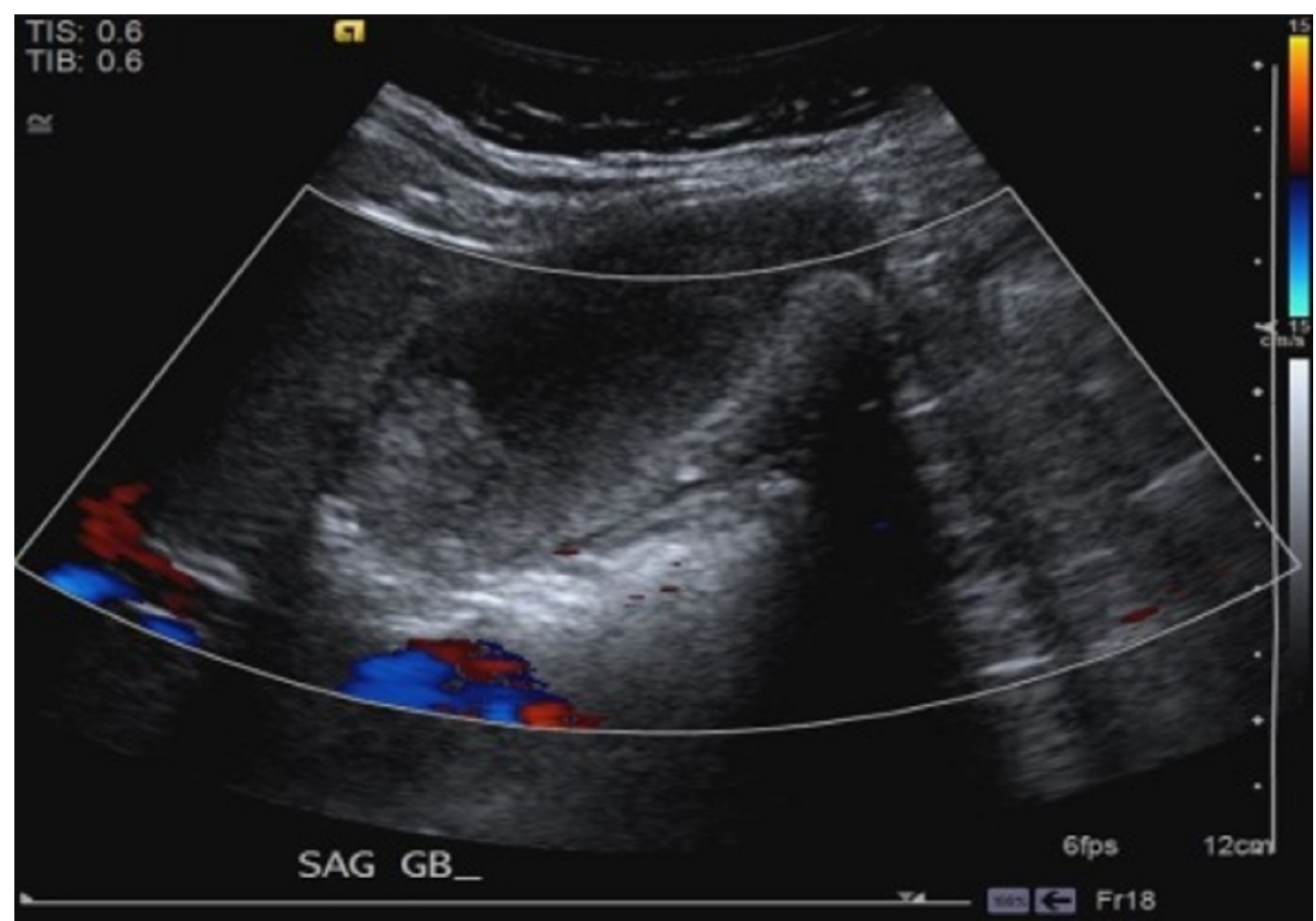

FIGURE 2: Initial right upper quadrant ultrasound.

The image demonstrates no discrete internal flow within the gallbladder with blood flow in the adjacent vasculature. 


\section{Cureus}

The patient was followed closely by her PCP until her symptoms resolved and elevated liver enzymes normalized over the next few months. No additional imaging was performed at that time, and no surgery was scheduled.

In April 2014, the patient developed RUQ pain that worsened over the next four months, prompting her to seek medical help. In August 2014, she was reevaluated by her PCP and subsequently underwent an acute abdominal series (AAS), RUQ ultrasound, and nuclear medicine hepatobiliary (HIDA) study. The ultrasound demonstrated cholelithiasis and echogenic sludge within the gallbladder with hypoechoic and hyperemic adjacent liver parenchyma. A mildly enlarged common duct was also observed. These findings were concerning for chronic cholecystitis, xanthogranulomatous cholecystitis, or early acute cholecystitis (Figure 3). The HIDA scan results were also consistent with acute cholecystitis (Figure 4).

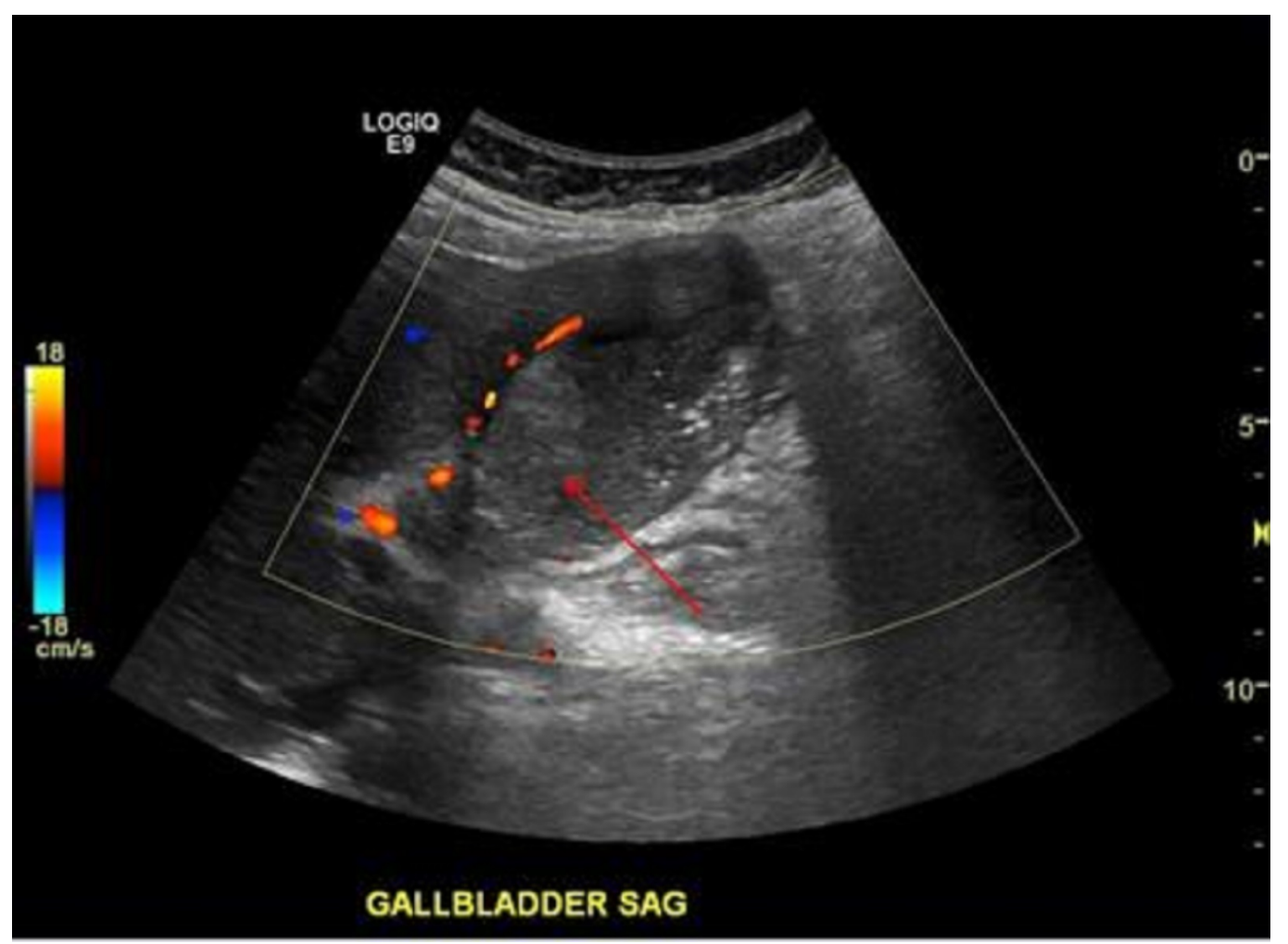

\section{FIGURE 3: Right upper quadrant ultrasound.}

The image demonstrates sludge/stone like material within the lumen of the gallbladder (red arrow) with a rim of hyperemia initially interpreted as outside of the gallbladder given the clinical appearance of acute cholecystitis. 


\section{Cureus}

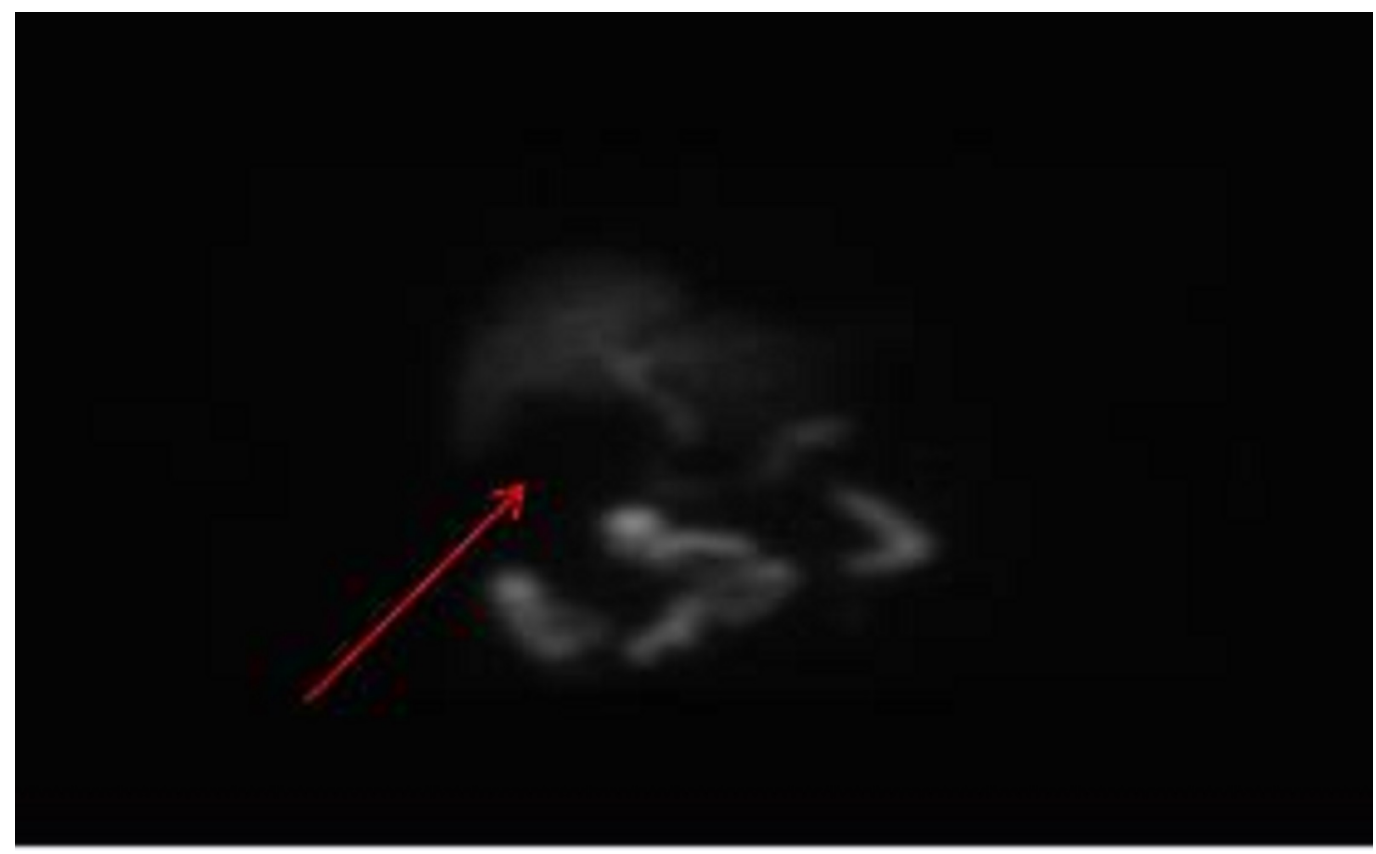

FIGURE 4: HIDA scan.

The image demonstrates excretion through the biliary system into the duodenojejunal junction (DJJ), but with no uptake within the gallbladder/cystic duct (red arrow).

The patient was taken to surgery for a laparoscopic cholecystectomy for suspected cholecystitis. The laparoscopic approach was converted to an open procedure after the discovery of a mass within the lumen of the gallbladder, a second mass near the hepatic flexure of the colon, and enlarged portal vein lymph nodes. During the operation, the patient underwent a radical cholecystectomy, right hemicolectomy with primary anastomosis, and a portal vein lymph node dissection (LND). Pathology of the gallbladder mass demonstrated infiltrating papillary adenocarcinoma invading the perimuscular connective tissue. The pathology also revealed one portocaval lymph node that was positive for adenocarcinoma. The patient's stage of disease was determined to be IVB (pT2N2). After surgery, the patient underwent radiotherapy to $4500 \mathrm{cGy}$, targeting the gallbladder fossa and the involved lymph nodes, with concurrent chemotherapy (capecitabine $625 \mathrm{mg} / \mathrm{m} 2$ twice daily). Abdominal CT and MRI scans were performed eight months after the completion of chemoradiation. At that time, there was no radiologic evidence of disease recurrence. Currently, the patient is fourteen months post treatment. Overall, she is doing well and continues to receive close follow-up care.

\section{Discussion}

GBC is an infrequent neoplasm that is associated with a high rate of regional lymph node metastasis and mortality $[2,6,9]$. Adjuvant chemoradiation is commonly recommended for node-positive or incompletely resected disease [7-8]. The extent of the resection correlates with survival. R0 resection is the most important because patients who undergo R2 resections do poorly in spite of chemoradiation. However, there are some long-term survivors in patients treated with adjuvant chemoradiation status post R0/R1 resections [6,9-10]. Therefore, early detection is important because patients found at stage T1N0 would have a greater chance for surgical cure and spare them the potential toxicity of adjuvant therapy.

Early diagnosis can be difficult because symptoms can mimic or be caused by coexisting cholecystitis, which is a common condition [5]. Therefore, screening patients who present with 


\section{Cureus}

these symptoms is essential given the possibility for a coexisting GBC. Symptoms early in the disease process can also be vague, often leading to a delay in diagnosis. The most common complaint in the symptomatic patient with GBC is RUQ pain, specifically in the right hypochondrium. Other warning signs, which our patient did not exhibit, include weight loss, anorexia, nausea and/or vomiting, jaundice, and pruritus [11-12]. Routine laboratory tests are generally nondiagnostic and do not significantly improve the identification of GBC preoperatively, as exemplified by our case [12]. Serum tumor markers, carcinoembryonic antigen (CEA), and carbohydrate antigen 19-9 (CA 19-9) are frequently elevated in patients with GBC, but are not useful in its diagnosis because of their lack of sensitivity and specificity [13-14].

Imaging with ultrasound and CT has improved preoperative diagnosis of GBC. Despite these advancements, only 50\% of gallbladder cancers are recognized before surgery [12]. Ultrasound is often the initial imaging study of choice for patients presenting with symptoms consistent with gallstone disease. The RUQ ultrasound is perhaps the single most important test in helping lead to the diagnosis of GBC preoperatively. Ultrasound images from a group of patients diagnosed with GBC incidentally were reviewed retrospectively and found to have suspicious findings on reevaluation [15].

The most common ultrasound findings include calcified and echogenic mucosal masses, which can be associated with cholelithiasis or porcelain gallbladder [15]. High-risk features on ultrasonography also include solitary or displaced gallstone, intraluminal mass, gallbladderreplacing or invasive mass, and discontinuity of the mucosal echo [15]. Other findings that are suggestive of GBC include the loss of the interface between the gallbladder and liver or direct liver infiltration [15]. Moreover, ultrasound abnormalities are often more subtle in early stage disease, making detection more challenging [15]. If abnormalities or suspicious findings are detected on ultrasound, further evaluation with other non-invasive imaging is warranted. Consideration of GBC in the differential diagnosis may help to improve detection before surgery (Figures 5-7), potentially leading to the discovery of the disease in its early stages.

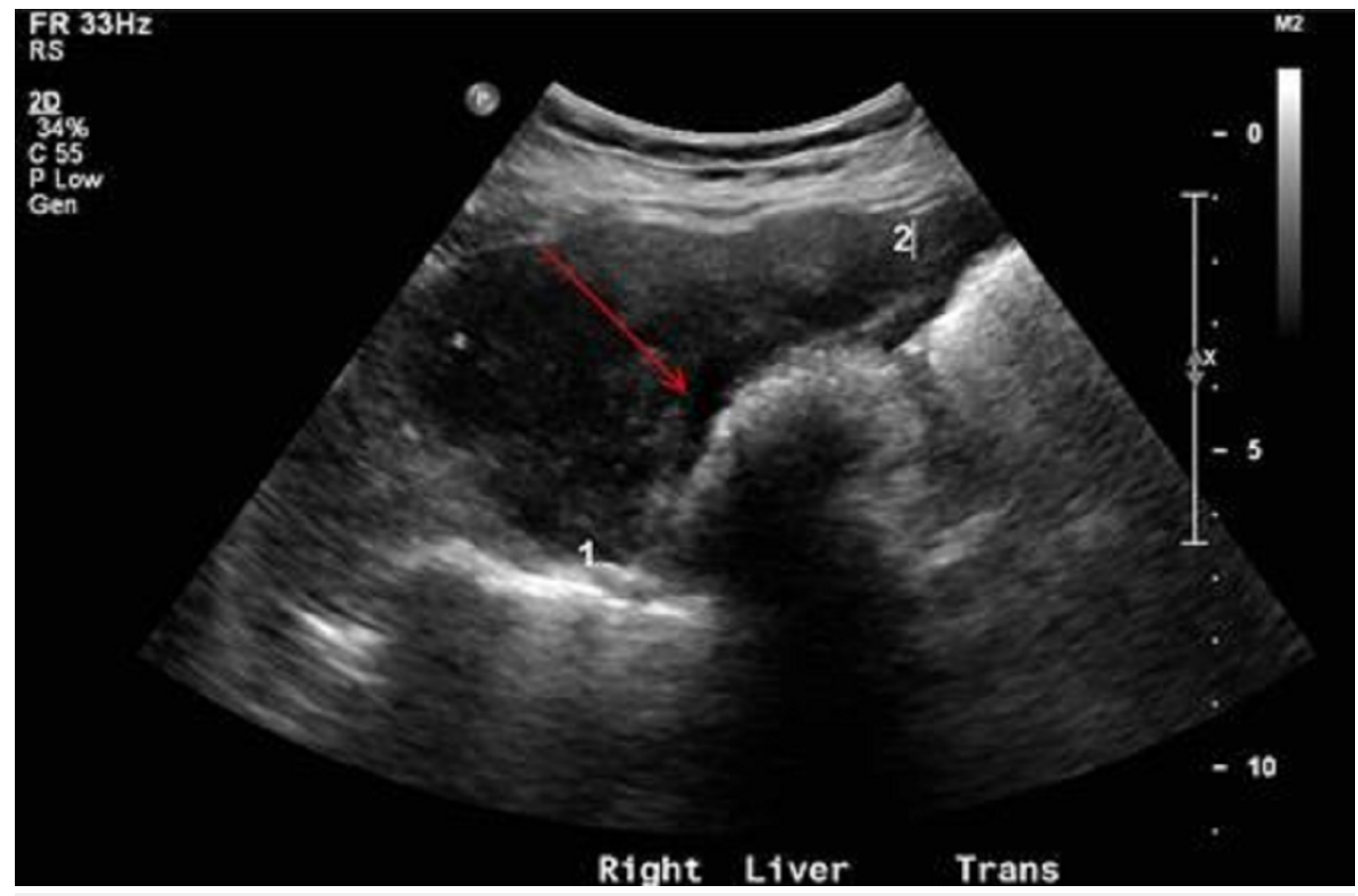

FIGURE 5: Companion case. Right upper quadrant ultrasound of a patient who presented a few months after the first patient. 


\section{Cureus}

The image demonstrates a hypoechoic lesion near the expected location of the gallbladder (red arrow). Given the suspicious ultrasound finding and recent prior GBC case, leading differential diagnosis was neoplasm. As a result, further evaluation with a CT scan was recommended.

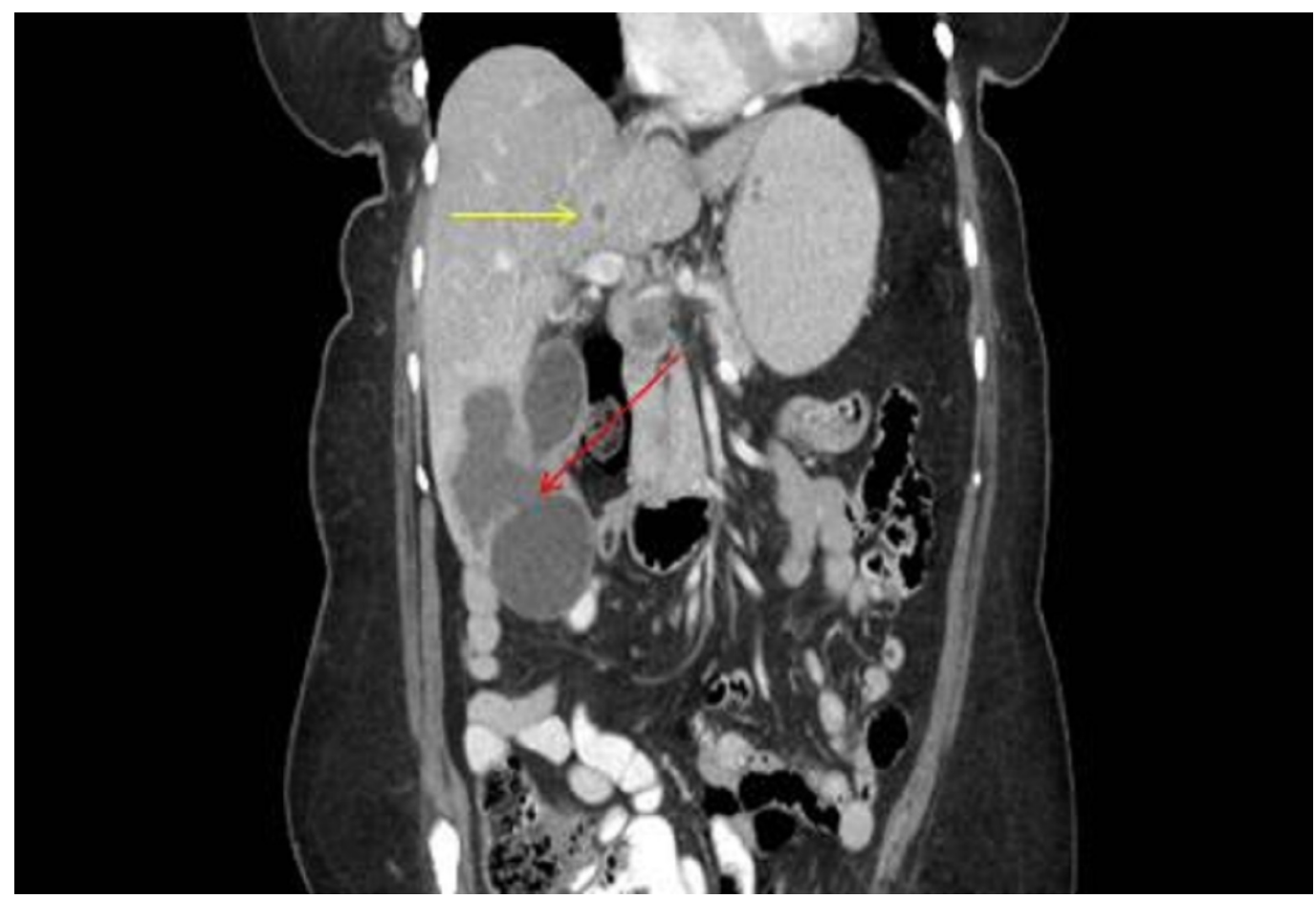

FIGURE 6: CT scan.

The image demonstrates a coronal CT showing a hypodense mass with direct invasion into the right hepatic lobe (red arrow), with additional small lesion in the left hepatic lobe (yellow arrow). Not shown is portohepatous and peripancreatic lymphadenopathy. The extent of disease suggests an element of patient denial of symptoms prior to clinical presentation.

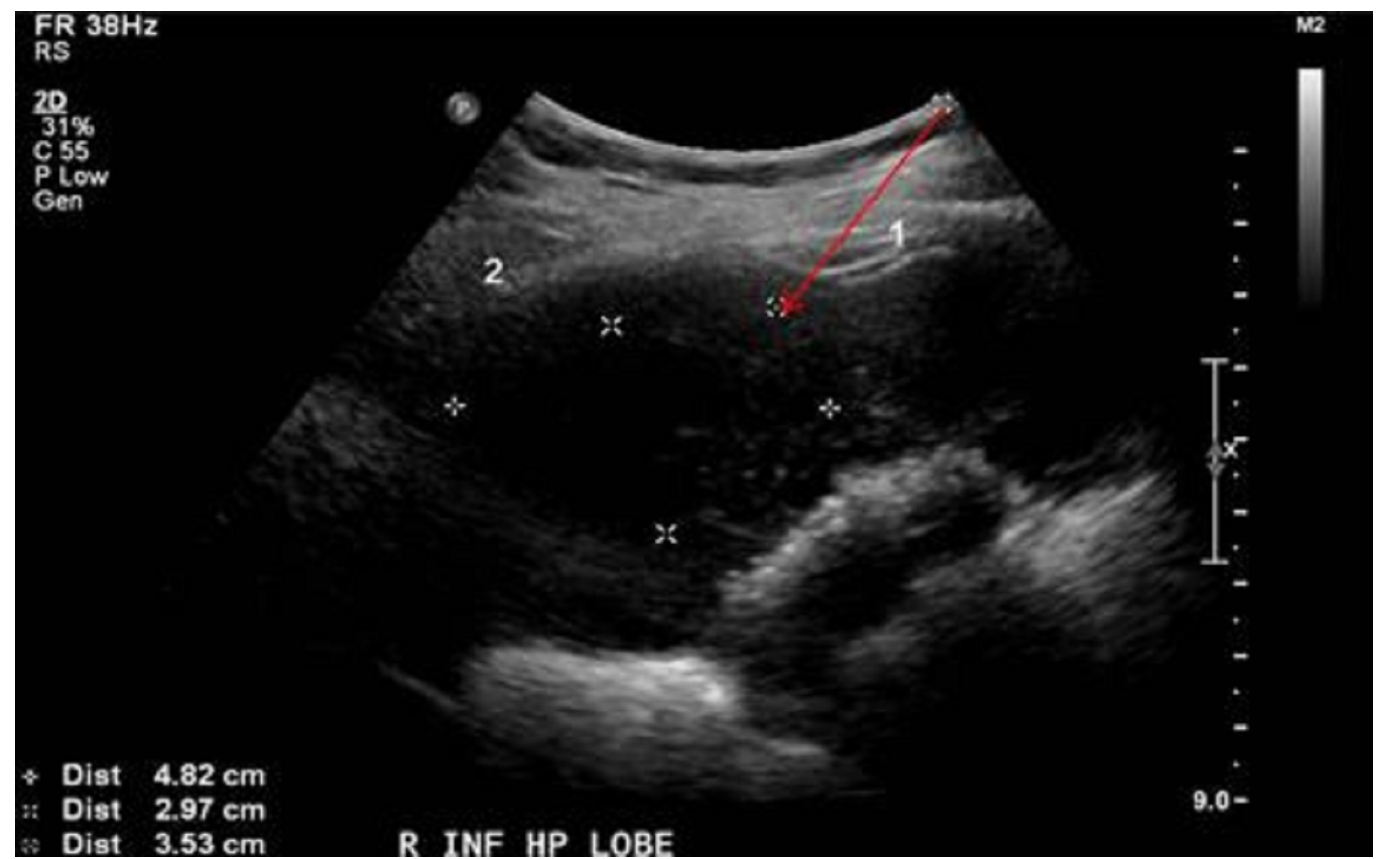


FIGURE 7: Preparatory imaging of ultrasound guided biopsy in the companion case.

The image demonstrates the target lesion and the planned route of the needle (red arrow). The biopsy results revealed a primary biliary cancer, most likely gallbladder carcinoma.

The most useful, non-invasive imaging studies for evaluating GBC preoperatively include CT, magnetic resonance imaging (MRI), and/or magnetic resonance cholangiopancreatography (MRCP) (Figures 5, 6). CT has been shown to be useful in defining the extent of GBC and in determining the resectability in advanced stages [16-17]. Additionally, CT has a low to moderate sensitivity for detecting gastrointestinal, omental, and abdominal wall involvement, but because of its high positive predictive value in detecting liver invasion, lymph node involvement, and/or distant metastases, it remains a useful imaging study in GBC preoperatively [16-17]. MRI and MRCP have also been shown to be useful in preoperative staging of GBC with a high sensitivity in identifying hepatic invasion and lymph node metastasis [18]. Biopsies of the detected gallbladder masses are often performed via ultrasound guidance (Figure 7), but can also be performed under CT guidance, endoscopic ultrasound guidance, endoscopic retrograde cholangiopancreatography (ERCP), or by laparoscopy.

Regarding further procedures and tests for GBC, endoscopic ultrasound (EUS) is a minimally invasive procedure that has been shown to be more accurate than RUQ ultrasound at imaging the gallbladder. EUS is also helpful in the differential diagnosis of gallbladder polyps and excellent in staging tumor depth [19-20]. ERCP and PET are two less commonly used imaging modalities used for evaluating GBC preoperatively. Generally, PET is not widely used prior to surgical resection because of its low sensitivity for detecting extrahepatic metastases, especially in patients with peritoneal carcinomatosis [21]. Although PET/CT is not commonly used before surgical resection, several studies have shown a potential benefit in detecting distant metastasis [21-24]. One retrospective study demonstrated that in patients with potentially resectable tumors based on conventional imaging, PET imaging was able to detect occult metastatic disease and ultimately changed the management in almost twenty-five percent of the patients [24].

\section{Conclusions}

Our case illustrates the challenge of preoperative, early-stage GBC diagnosis. Despite multiple imaging studies, GBC was not diagnosed in the described patient until laparoscopy for acute cholecystitis. In the companion case, GBC was advanced at the time of imaging, which may have been due to patient denial of symptoms. Given that early detection is important in both decreasing the morbidity and mortality of GBC, with potential for surgical cure in cases limited to the gallbladder, patients with RUQ ultrasound findings such as gallstones or polyps should be offered surgical consultation. Further, consideration of GBC within the differential diagnosis by primary care clinicians, radiologists, and surgeons may serve to maximize discovery before the time of surgery.

"The views expressed in this case report are those of the authors and do not reflect the official policy of the Department of Army/Navy/Air Force, Department of Defense, or U.S. Government."

\section{Additional Information}




\section{Disclosures}

Human subjects: All authors have confirmed that this study did not involve human participants or tissue. Conflicts of interest: In compliance with the ICMJE uniform disclosure form, all authors declare the following: Payment/services info: All authors have declared that no financial support was received from any organization for the submitted work. Financial relationships: All authors have declared that they have no financial relationships at present or within the previous three years with any organizations that might have an interest in the submitted work. Other relationships: All authors have declared that there are no other relationships or activities that could appear to have influenced the submitted work.

\section{References}

1. Carriaga MT, Henson DE: Liver, gallbladder, extrahepatic bile ducts, and pancreas. Cancer. 1995, 75:171-190. 10.1002/1097-0142(19950101)75:1+<171::AIDCNCR2820751306>3.0.CO;2-2

2. Duffy A, Capanu M, Abou-Alfa GK, et al: Gallbladder cancer (GBC): 10-year experience at Memorial Sloan-Kettering Cancer Centre (MSKCC). J Surg Oncol. 2008, 98:485-489. 10.1002/jso.21141

3. Zatonski WA, Lowenfels AB, Boyle P, et al: Epidemiologic aspects of gallbladder cancer: a case-control study of the SEARCH Program of the International Agency for Research on Cancer. J Natl Cancer Inst. 1997, 89:1132-1138. 10.1093/jnci/89.15.1132

4. Rustagi T, Dasanu CA: Risk factors for gallbladder cancer and cholangiocarcinoma: similarities, differences and updates. J Gastrointest Cancer. 2012, 43:137-147. 10.1007/s12029-011-9284-y

5. Hsing AW, Gao YT, Han TQ, et al: Gallstones and the risk of biliary tract cancer: a populationbased study in China. Br J Cancer. 2007, 97:1577-1582. 10.1038/sj.bjc.6604047

6. Groot Koerkamp B, Fong Y: Outcomes in biliary malignancy. J Surg Oncol. 2014, 110:585-591. 10.1002/jso.23762

7. Horgan AM, Amir E, Walter T, Knox JJ: Adjuvant therapy in the treatment of biliary tract cancer: a systematic review and meta-analysis. J Clin Oncol. 2012, 30:1934-1940. $10.1200 /$ jco.2011.40.5381

8. Wang SJ, Lemieux A, Kalpathy-Cramer J, et al: Nomogram for predicting the benefit of adjuvant chemoradiotherapy for resected gallbladder cancer. J Clin Oncol. 2011, 29:46274632. 10.1200/jco.2010.33.8020

9. Cariati A, Piromalli E, Cetta F: Gallbladder cancers: associated conditions, histological types, prognosis, and prevention. Eur J Gastroenterol Hepatol. 2014, 26:562-569. 10.1097/meg.0000000000000074

10. Pilgrim CH, Groeschl RT, Turaga KK, Gamblin TC: Key factors influencing prognosis in relation to gallbladder cancer. Dig Dis Sci. 2013, 58:2455-2462. 10.1007/s10620-013-2713-y

11. Misra S, Chaturvedi A, Misra NC, Sharma ID: Carcinoma of the gallbladder. Lancet Oncol. 2003, 4:167-176. 10.1016/S1470-2045(03)01021-0

12. Löhe F, Meimarakis G, Schauer C, Angele M, Jauch KW, Schauer RJ: The time of diagnosis impacts surgical management but not the outcome of patients with gallbladder carcinoma. Eur J Med Res. 2009, 14:345-351.

13. Strom BL, Maislin G, West SL, et al: Serum CEA and CA 19-9: potential future diagnostic or screening tests for gallbladder cancer. Int J Cancer. 1990, 45:821-824. 10.1002/ijc.2910450505

14. Ritts RE Jr, Nagorney DM, Jacobsen DJ, Talbot RW, Zurawski VR Jr: Comparison of preoperative serum CA19-9 levels with results of diagnostic imaging modalities in patients undergoing laparotomy for suspected pancreatic or gallbladder disease. Pancreas. 1994, 9:707716.

15. Wibbenmeyer LA, Sharafuddin MJ, Wolverson MK, Heiberg EV, Wade TP, Shields JB: Sonographic diagnosis of unsuspected gallbladder cancer: imaging findings in comparison with benign gallbladder conditions. AJR Am J Roentgenol. 1995, 165:1169-1174. 10.2214/ajr.165.5.7572497

16. Kumar A, Aggarwal S: Carcinoma of the gallbladder: CT findings in 50 cases . Abdom Imaging. 1994, 19:304-308. 10.1007/BF00198184 
17. Ohtani T, Shirai Y, Tsukada K, Muto T, Hatakeyama K: Spread of gallbladder carcinoma: CT evaluation with pathologic correlation. Abdom Imaging. 1996, 21:195-201.

10.1007/s002619900045

18. Schwartz LH, Black J, Fong Y, et al: Gallbladder carcinoma: findings at MR imaging with MR cholangiopancreatography. J Comput Assist Tomogr. 2002, 26:405-410. 10.1097/00004728200205000-00015

19. Sugiyama M, Atomi Y, Yamato T: Endoscopic ultrasonography for differential diagnosis of polypoid gall bladder lesions: analysis in surgical and follow up series. Gut. 2000, 46:250-254. 10.1136/gut.46.2.250

20. Sadamoto Y, Kubo H, Harada N, Tanaka M, Eguchi T, Nawata H: Preoperative diagnosis and staging of gallbladder carcinoma by EUS. Gastrointest Endosc. 2003, 58:536-541. 10.1067/S0016-5107(03)01961-8

21. Anderson CD, Rice MH, Pinson CW, Chapman WC, Chari RS, Delbeke D: Fluorodeoxyglucose PET imaging in the evaluation of gallbladder carcinoma and cholangiocarcinoma. J Gastrointest Surg. 2004, 8:90-97. 10.1016/j.gassur.2003.10.003

22. Petrowsky H, Wildbrett $\mathrm{P}$, Husarik DB, et al: Impact of integrated positron emission tomography and computed tomography on staging and management of gallbladder cancer and cholangiocarcinoma. J Hepatol. 2006, 45:43-50. 10.1016/j.jhep.2006.03.009

23. Lee SW, Kim HJ, Park JH, et al: Clinical usefulness of 18F-FDG PET-CT for patients with gallbladder cancer and cholangiocarcinoma. J Gastroenterol. 2010, 45:560-566. 10.1007/s00535-009-0188-6

24. Corvera CU, Blumgart LH, Akhurst T, et al: 18F-fluorodeoxyglucose positron emission tomography influences management decisions in patients with biliary cancer. J Am Coll Surg. 2008, 206:57-65. 10.1016/j.jamcollsurg.2007.07.002 